Trauma Berufskrankh 2007 - 9 [Suppl 2]:S149-S153 DOI 10.1007/s10039-006-1127-x

Online publiziert: 11. April 2006

๑) Springer Medizin Verlag 2006

A. Seekamp

Klinik für Unfallchirurgie (Traumatologie), Universitätsklinikum

Schleswig-Holstein, Campus Kiel, Kiel

\title{
Externe Fixation am Beckenring
}

\section{Notfallstabilisierung des Beckenrings durch externe Fixation}

Durch die Notfallstabilisierung des Beckenrings soll das intrapelvine Volumen reduziert werden [5].

\section{Konservativ}

Am einfachsten kann die Stabilisierung durch eine Tuchumlegung des Beckenrings erfolgen. Noch effektiver ist eine pneumatische Beckenkompression. Beide Maßnahmen können im Bereich der präklinischen Versorgung angewendet werden oder aber in Situationen, in denen operative Verfahren nicht zur Verfügung stehen.

\section{Supraazetabulärer Fixateur externe}

Experimentelle Untersuchungen haben gezeigt, dass seine Anlage zu einer guten Reduzierung des intrapelvinen Beckenvolumens führen kann, jedoch wird keine ausreichende mechanische Stabilität des hinteren Beckenrings erreicht [3]. Daher ist insbesondere bei Typ-C-Beckenringfrakturen die Beckenzwinge indiziert und führt zu der bestmöglichen Stabilisierung des gesamten Beckenrings im Vergleich zu den zuvor genannten Methoden [2].

\section{Wahl des Stabilisierungsverfahrens}

Im Rahmen der Leitlinienerstellung für das Vorgehen bei komplexen Beckenfrakturen hat sich herauskristallisiert, dass der Fixateur externe als stabilisierende
Maßnahme für den vorderen Beckenring in jedem Fall ausreichend ist, bei Typ-CFrakturen allerdings eine zusätzliche hintere Kompression benötigt wird. Die mechanische Stabilisierung des hinteren Beckenrings ist durch den Fixateur externe allein nicht suffizient zu erreichen [9]. Keine evidente Aussage gibt es bisher bezüglich der hämodynamischen Stabilisierung für die beiden operativen Stabilisierungsmöglichkeiten.

\section{Anlage der Beckenzwinge}

Sie ist als notfallchirurgischer Eingriff zu werten und sollte entweder bereits im Rahmen des Schockraummanagements vorgenommen werden oder aber spätestens als Erstmaßnahme im Operationssaal. Da sie rasch zu erfolgen hat, muss man sich zuvor mit dem Implantat vertraut machen und die Applikation im Vorfeld mehrfach geübt haben. Das Anlegen der Beckenzwinge am Patienten sollte unter sterilen Bedingungen erfolgen. Wichtigster Schritt ist die Identifikation der Fixationspunkte (• Abb. 1). Diese liegen beidseits in dem gedachten Kreuzpunkt einer Linie in Verlängerung des Femurschafts mittig über dem Trochanter major sowie einer hierzu nahezu senkrecht stehenden Linie von der Spina iliaca superior anterior zur Spina iliaca superior posterior. In dieser Höhe erfolgt ein Hautschnitt. Mit der Schere wird bis auf die Beckenschaufel vorpräpariert und die Beckenschaufel getastet. Der Eintrittspunkt liegt genau im Bereich des Übergangs von der schrägen Kortikalis der Beckenschaufel zur eher senkrecht ste- 


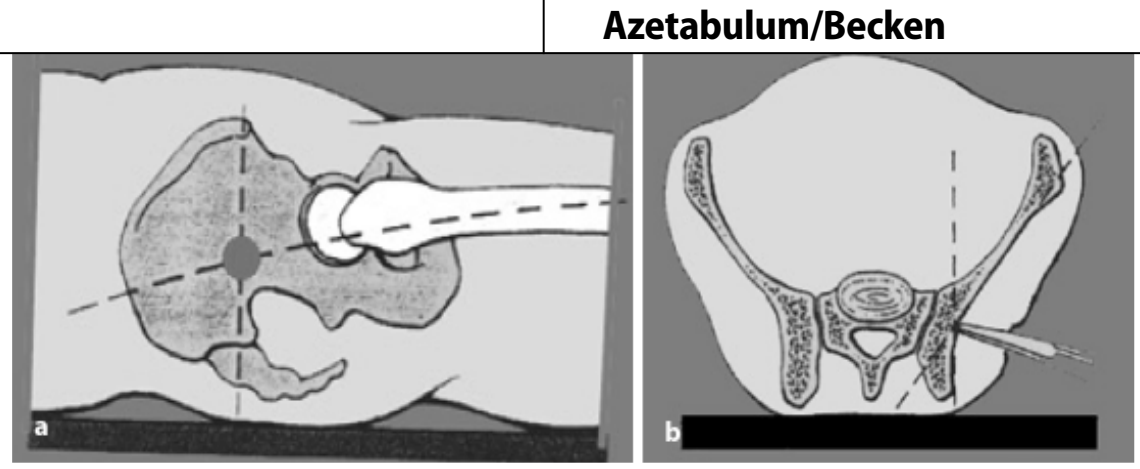

Abb. 1 ॥ Identifikation des maximalen Druckpunkts für die Pins der Beckenzwinge
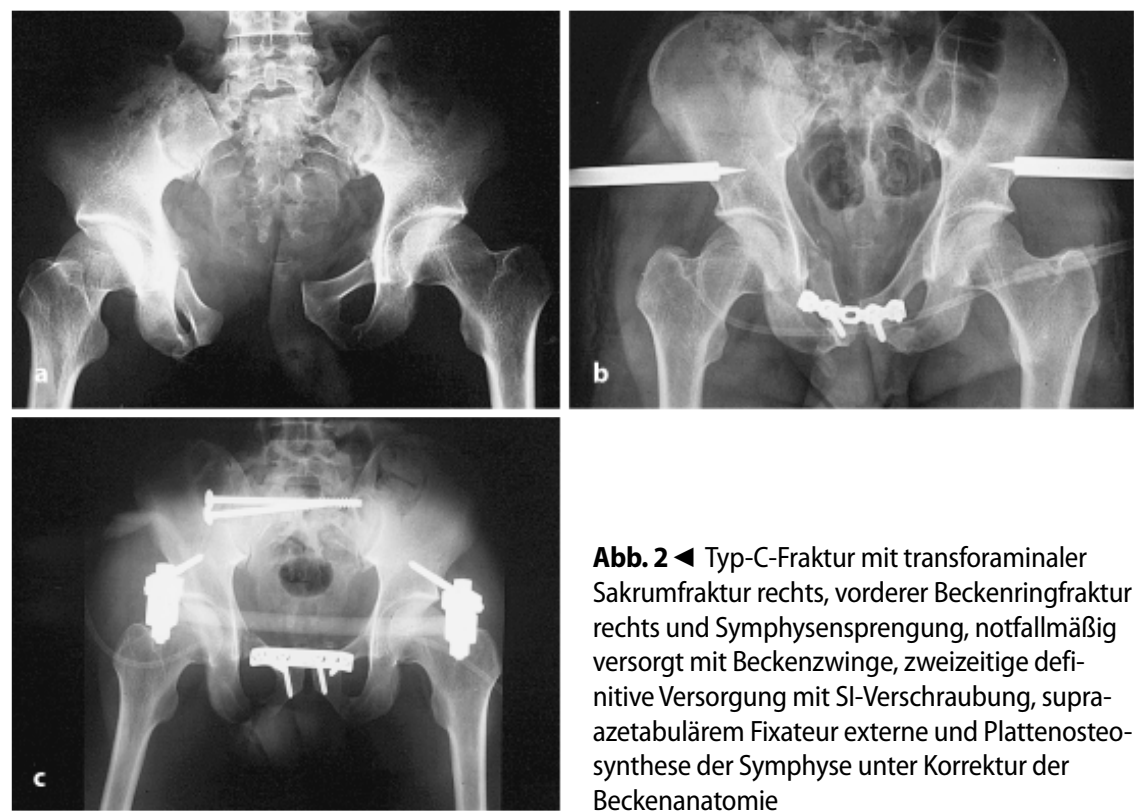

Abb. 24 Typ-C-Fraktur mit transforaminaler Sakrumfraktur rechts, vorderer Beckenringfraktur rechts und Symphysensprengung, notfallmäßig versorgt mit Beckenzwinge, zweizeitige definitive Versorgung mit SI-Verschraubung, supraazetabulärem Fixateur externe und Plattenosteosynthese der Symphyse unter Korrektur der Beckenanatomie
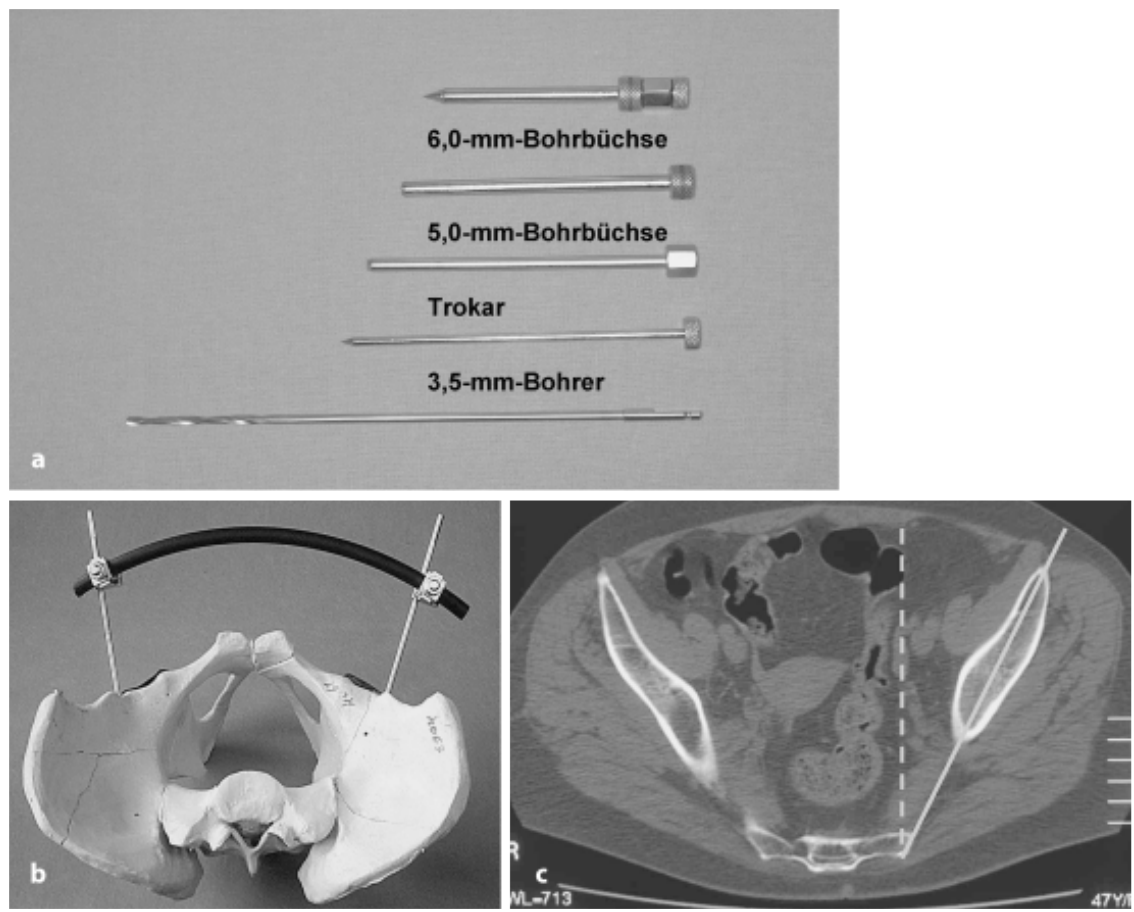

Abb. 3 \ Anbringen des supraazetabulären Fixateur externe, a notwendige Bohrhülsen, b optimale Lage am Modell, c axialer CT-Schnitt henden Kortikalis in Höhe des Sakrums. Nach dieser Präparation werden beidseits gleichzeitig der entsprechende Pin eingebracht und anschließend die Beckenzwinge aufgesetzt. Ziel der notfallmäßigen Anlage der Beckenzwinge ist keine anatomische Reposition des Beckenrings, sondern lediglich eine Kompression des hinteren Beckenrings.

Bei dem in $\bullet$ Abb. 2 dargestellten Beispielfall wurde im Rahmen einer Unterbauchlaparotomie gleichzeitig die Symphyse mit einer Rekonstruktionsplatte fixiert. Nach der Operation erschien jedoch der Beckenring insgesamt etwas zu klein. Nachdem sich der Patient im weiteren intensivstationären Aufenthalt stabilisiert hatte, wurde der Beckenring zweizeitig anatomische rekonstruiert, indem er nach Abnehmen der Beckenzwinge durch eine 2 fache transiliosakrale Verschraubung fixiert wurde. Auch im Bereich der Symphyse wurde die Rekonstruktionsplatte durch eine schmale $4,5 \mathrm{er}$ Platte ausgetauscht und die vordere Beckenringfraktur zusätzlich mit einem supraazetabulären Fixateur gesichert.

\section{Retrospektive Analyse der AG Becken I der DGU}

In ihr wurden insgesamt 1076 Patienten mit isolierter Beckenringfraktur dokumentiert [4]. Bei etwas über 10\% derselben erfolgte eine Notfallstabilisierung, wofür ausschließlich Typ-B- und -C-Frakturen in Betracht kamen, die einen Gesamtanteil von 19\% ausmachten. Jeder 5. Patient mit einer Typ-B- oder C-Fraktur benötigte eine notfallmäßige Stabilisierung. Im Rahmen derer wurde bei 19 Patienten die Beckenzwinge angelegt, bei 48 Patienten der Fixateur externe, und bei 42 Patienten wurde bereits initial eine definitive interne Fixation vorgenommen.

\section{Definitive Versorgung durch externe Fixation}

Die definitive operative Frakturversorgung richtet sich nach dem Prinzip, dass jeweils eine residual bestehende Instabilität am Ort ihrer Lokalisation stabilisiert werden soll. Prinzipiell lassen sich 6 verschiedene Lokalisationen einer Insta- 
bilität unterscheiden (transsakral, transiliosakral, transiliakal, transazetabulär, transpubisch, transsymphysär). Im Bereich des Sakrums wird noch weiter unterteilt nach zentral, transforaminal und lateral.

\section{Supraazetabulärer Fixateur externe}

Indikationen. Sie bestehen in erster $\mathrm{Li}^{-}$ nie für die so genannten transpubischen Instabilitäten, wobei es sich in erster Linie um vordere Beckenringfrakturen handelt [10], und zwar in der überwiegenden Zahl der Fälle um Typ-B-Frakturen, die durch eine laterale Kompression des Beckenrings entstanden sind. Der Fixateur externe dient hierbei als so genannte Distanzosteosynthese [1]. Eine alleinige oder parallel bestehende Ruptur der Symphyse wird besser mit einer direkten Plattenosteosynthese versorgt. Je nach Grad der rotatorischen Instabilität kann in Ausnahmefällen auch eine gleichzeitige Stabilisierung des hinteren Beckenrings erforderlich sein.

Eine weitere Indikation ist die transpubische Instabilität des Beckenrings im Rahmen von Typ-C-Frakturen. Da es sich hierbei definitionsgemäß um eine gleichzeitig bestehende vertikale Instabilität des hinteren Beckenrings handelt, kann der Fixateur externe nur in Kombination mit einer Stabilisierung desselben angewendet werden. Für eine ggf. gleichzeitig bestehende Symphysenruptur gilt Gleiches wie bei den Typ-B-Frakturen.

Auch bei Beckenfrakturen im Kindesalter ist der Fixateur externe indiziert, wobei die Indikation im Allgemeinen noch weiter gestellt wird als im Erwachsenenalter.

\section{Anlage des supraazetabulären Fixateur} externe. Es werden lediglich 2 SchanzSchrauben von $4,5 \mathrm{~mm}$ oder $5 \mathrm{~mm}$ Durchmesser verwendet, sowie 2 Fixationsbacken und ein entsprechender gebogener oder gerader Fixationsbügel (• Abb.3). Der Eintrittspunkt für die Schanz-Schraube liegt etwas kranial der Spina iliaca anterior inferior [7]. Zur Identifikation wird zunächst die Spina iliaca anterior superior aufgesucht, die Stichinzision erfolgt an einem Punkt, der etwa 2 Querfinger von der Spina aus gese-

Trauma Berufskrankh 2007 - 9 [Suppl 2]:S149-S153 DOI 10.1007/s10039-006-1127-x

(c) Springer Medizin Verlag 2006

\section{A. Seekamp \\ Externe Fixation am Beckenring}

\section{Zusammenfassung}

Eine externe Beckenringfixation kann sowohl im Rahmen einer Notfallstabilisierung als auch im Rahmen der definitiven Therapie einer Beckenringverletzung indiziert sein. Bei der Notfallstabilisierung hängt die Art der Fixation von der Klassifikation der Beckenfraktur ab. Der Fixateur externe ist als stabilisierende Maßnahme für den vorderen Beckenring ausreichend, bei Typ-C-Frakturen wird zusätzlich eine hintere Kompression benötigt. Die mechanische Stabilisierung des hinteren Beckenrings ist durch den Fixateur externe allein nicht suffizient zu erreichen, hier ist die Beckenzwinge indiziert. Ihre Anlage ist

\section{External fixation in the pelvic ring}

\section{Abstract}

External fixation of the pelvic ring can be indicated both for emergency stabilization and as part of the definitive treatment of an injury to the pelvic ring. In the case of emergency stabilization the type of fixation applied depends on how the pelvic fracture is classified. The external fixator is an adequate stabilizer for the anterior part of the pelvic ring, while for type $\mathrm{C}$ fractures posterior compression is needed in addition. Adequate mechanical stabilization of the posterior part of the pelvic ring cannot be achieved by means of the external fixator alone, and in these cases a pelvic clamp is indicated. Its application must be regarded as an emergency surgical proce- als notfallchirurgischer Eingriff zu werten. Im Rahmen der definitiven Stabilisierung ist bei allen Typ-B-Frakturen der supraazetabulär angebrachte Fixateur externe eine gute Lösung Bei Typ-C-Frakturen kann er als zusätzliche definitive Stabilisierung dienen, aufgrund der bei diesem Frakturtyp bestehenden Instabilität des hinteren Beckenrings ist dessen gleichzeitige interne Fixation erforderlich.

\section{Schlüsselwörter}

Beckenfraktur · Beckenringfixation · Klassifikation - Supraazetabulärer Fixateur externe Beckenzwinge

dure. When it is part of the definitive stabilization process, the external fixator applied in a supra-acetabular position is a good solution in all type $\mathrm{B}$ fractures. It can also be used for type $C$ fractures for additional definitive stabilization, but because of the instability of the posterior pelvic ring that is always present in the case of this type of fracture simultaneous internal fixation is also required.

\section{Keywords}

Pelvic fracture - Pelvic ring fixation - Classification - Supra-acetabular fixateur externe · Pelvic clamp 


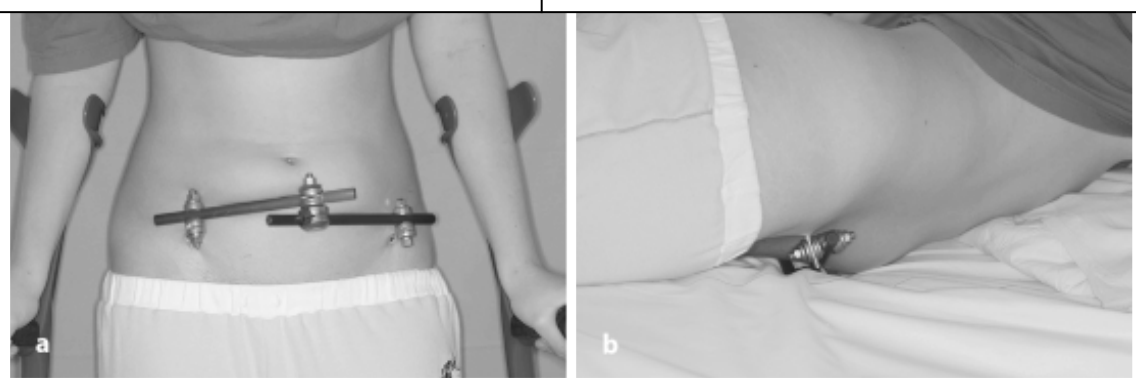

Abb. 4 \ Gute Toleranz des Fixateur externe unter Mobilisierung (a), selbst Liegen auf dem Bauch (b) erscheint ohne Beschwerden möglich
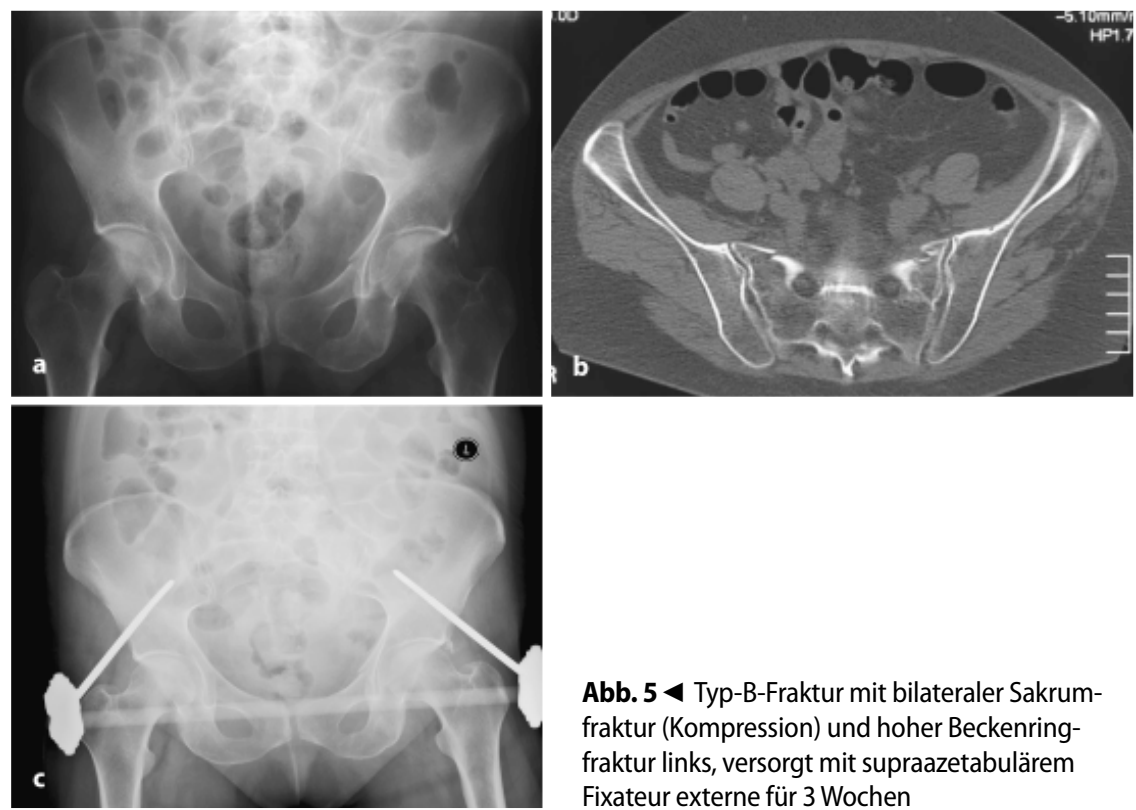

Abb. 54 Typ-B-Fraktur mit bilateraler Sakrumfraktur (Kompression) und hoher Beckenringfraktur links, versorgt mit supraazetabulärem Fixateur externe für 3 Wochen
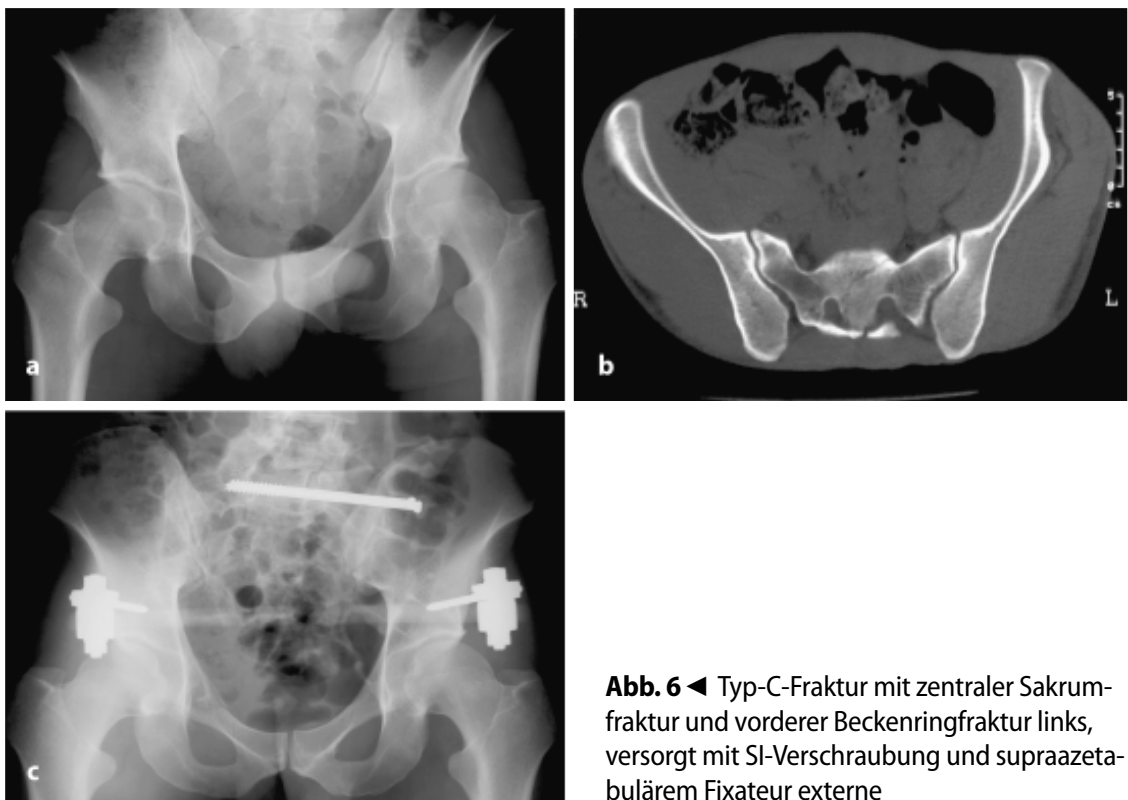

hen nach innen liegt und 2 Querfinger nach kaudal. Nach Hautinzision wird die Faszie mit der Schere bis in die Tiefe gespalten und der Eintrittspunkt am Beck- und die Kortikalis mit dem 3,5-mm-Bohrer eröffnet. Die Schanz-Schraube wird aufgesetzt und bei etwa $10^{\circ}$ Neigung nach kranial und etwa $30^{\circ}$ Neigung nach medial eingeschraubt. Hierbei kann die volle Breite der Beckenschaufel bis zur Spina iliaca posterior inferior ausgenutzt werden. Die richtige Position der Lage kann in a.-p. Projektion sowie Inlet- und Outlet-Technik mit dem Bildwandler kontrolliert werden.

Ergebnisse. Normalerweise wird der supraazetabuläre Fixateur recht gut toleriert, und die Patienten können frühzeitig mobilisiert werden (- Abb. 4). Lediglich die Pinpflege sollte sorgfältig durchgeführt werden. Als Beispiel einer typischen Indikation ist in $\bullet$ Abb. 5 eine Typ-B-Fraktur mit einer beidseitigen lateralen Kompressionsfraktur gezeigt. Linksseitig liegen eine hohe vordere Beckenringfraktur sowie beidseits eine wenig dislozierte laterale Kompressionsfraktur der Pars lateralis des Sakrums vor.

In der Regel ist eine vordere Beckenringfraktur nach 3 Wochen verheilt. Im eigenen Vorgehen werden nach diesem Zeitraum zunächst der Fixationsbügel abgenommen und der Beckenring klinisch auf Stabilität geprüft. Werden von Seiten des Patienten keine Beschwerden angegeben, werden die Schanz-Schrauben ebenfalls komplett entfernt. Dieses Vorgehen gilt auch für Typ-C-Verletzungen, bei denen neben einer Stabilisierung des hinteren Beckenrings, z. B. durch eine iliosakrale Verschraubung [8], auch ein Fixateur externe zur Stabilisierung des vorderen Beckenrings angebracht wurde (• Abb. 6).

In der schon erwähnten Analyse der AG Becken I [3] wurden insgesamt 278 Beckenringfrakturen mit einer definitiven operativen Versorgung dokumentiert. Der Fixateur externe wurde innerhalb dieses Kollektivs in insgesamt 103 Fällen verwendet. Bei 51 Patienten $(18,3 \%)$ all dieser Frakturen wurde er als ausschließliche Stabilisierungstechnik eingesetzt. Innerhalb dieses Kollektivs war die Mehrzahl der Frakturen als TypB-Fraktur klassifiziert $(n=26)$, gefolgt von Typ-C-Frakturen mit 22 Fällen und TypA-Frakturen mit 3 Fällen. 


\section{Fazit}

Die externe Fixation am Beckenring erfolgt als Notfallstabilisierung bei Typ-CFrakturen mit einer Kreislaufinstabilität durch Beckenzwinge. Der Fixateur externe ist als alleinige Notfallstabilisierung bei Typ-B-Frakturen ausreichend, während er bei Typ-C-Frakturen gegenüber der Beckenzwinge weniger geeignet erscheint, aber ggf. zusätzlich eingesetzt werden kann.

Im Rahmen der definitiven Stabilisierung von Beckenringfrakturen stellt der supraazetabulär angebrachte Fixateur externe bei allen Typ-B-Frakturen eine gute Indikation dar. Dies gilt insbesondere für die transpubischen Instabilitäten, während Symphysenrupturen vorzugsweise mit einer Plattenosteosynthese versorgt werden sollten. Bei Typ-C-Frakturen kann er als zusätzliche definitive Stabilisierung dienen, dies gilt insbesondere, wie auch schon bei den Typ-B-Frakturen, für die transpubische Instabilität. Aufgrund der bestehenden Instabilität des hinteren Beckenrings bei diesem Frakturtyp ist dessen gleichzeitige interne Fixation in jedem Fall erforderlich.

\section{Korrespondierender Autor}

\section{Prof. Dr. A. Seekamp}

Klinik für Unfallchirurgie (Traumatologie), Universitätsklinikum Schleswig-Holstein, Campus Kiel Arnold-Heller-Straße 7, 24105 Kiel andreas.seekamp@unfchir.uni-kiel.de

Interessenkonflikt. Es besteht kein Interessenkonflikt. Der korrespondierende Autor versichert, dass keine Verbindungen mit einer Firma, deren Produkt in dem Artikel genannt ist, oder einer Firma, die ein Konkurrenzprodukt vertreibt, bestehen. Die Präsentation des Themas ist unabhängig und die Darstellung der Inhalte produktneutral.

\section{Literatur}

1. Bellabarba C, Ricci WM, Bollhofner BR (2000) Distraction external fixation in lateral compression pelvic fractures. J Orthop Trauma 14: 475-482

2. Ertl W, Keel M, Eid K et al. (2001) Control of severe hemorrhage using C-clamp and pelvic packing in multiply injured patients with pelvic ring disruption. J Orthop Trauma 15: 468-474

3. Pohlemann T, Krettek C, Hoffmann R et al. (1994) Biomechanischer Vergleich verschiedener Notfallstabilisierungsmaßnahmen am Beckenring. Unfallchirurg 97: 503-510

4. Pohlemann T, Gansslen A, Hartung S (1998) Beckenverletzungen. Hefte Unfallchirurg 266
5. Riemer BL, Butterfield SL, Diamond DL et al. (1993) Acute mortality associated with injuries to the pelvic ring: the role of early patient immobilization and external fixation. J Trauma 35: 671-677

6. Rommens PM, Hessmann MH (2002) Staged reconstruction of pelvic ring disruption: differences in morbidity, mortality, radiologic results, and functional outcomes between B1, B2/B3, and C-type lesions. J Orthop Trauma 16: 92-98

7. Rupp R, Ebraheim N, Jackson W (1994) Anatomic and radiographic considerations in the placement of anterior pelvic external pins. Clin Orthop 302: 213-218

8. Shuler TE, Boone DC, Gruen GS et al. (1995) Percutaneous iliosacral screw fixation: early treatment for unstable posterior pelvic ring disruptions. J Trauma 38: 453-458

9. Siegmeth A, Mullner T, Kukla C et al. (2000) Associated injuries in severe pelvic trauma. Unfallchirurg 103: 572-581

10. Tucker MC, Nork SE, Simonian PT (2000) Simple anterior pelvic external fixation. J Trauma 49: 989994 